\title{
Correlating dislocation behavior with macroscopic mechanical properties directly in the TEM through use of a novel tensile test device
}

\author{
K. Hattar,* J. J. Han, ${ }^{* *}$ T. Saif,** I. M. Robertson,* D. M. Follstaedt ${ }^{* * *}$, S. J. Hearne ${ }^{* * *}$ \\ Materials Science and Engineering, University of Illinois, Urbana IL 61801 \\ Mechanical and Industrial Engineering, University of Illinois, Urbana IL 61801 \\ *** Sandia National Laboratories, P. O. Box 5800, MS 1056 Albuquerque, NM 87185
}

High yield and fracture strength, low elongation and toughness, high strain-rate sensitivity, high strain-rate sensitivity index, low $\left(<10 \mathrm{~b}^{3}\right)$ activation volume, and high wear resistance are characteristic changes in properties of metals as the grain size decreases from large $(>1 \mu \mathrm{m})$, to ultrafine grained $(0.1 \mu \mathrm{m}<$ grain size $<1 \mu \mathrm{m})$, to nanograined $(<0.1 \mu \mathrm{m})$. The lack of strain hardening capacity, which is not compensated for by the increased strain-rate sensitivity index, makes these materials prone to inhomogeneous or localized deformation[1, 2] and accounts for their limited ductility. There remains considerable debate about the mechanisms responsible for these property changes as the grain size decreases. The change has been attributed to the deformation mechanisms changing from perfect dislocations, to partial dislocations, to twinning, and finally to grain boundary mediated process as the grain size decreases from micrometers to several tens of nanometers to a few nanometers. These transitions, identified largely from computer simulations, have not been validated experimentally.[3, 4] Previous in-situ TEM studies of strained metal films have claimed perfect dislocation activity, grain rotation, grain elongation and grain boundary mediated processes, although the transition grain sizes do not correspond to ones predicted by the computer simulations. [5, 6]

By using microlithographic techniques it is possible to fabricate miniature tensile test machines for operation in the TEM with varying degrees of functionality that permit observation of mechanisms and simultaneous measurement of the macroscopic properties.[7] An example of one such device is shown in Figs. 1a-b.[8] Although this device permits observation of the deformation processes and concurrent measurement of the macroscopic response, the device is operated in the TEM using a conventional displacement controlled straining stage. The metal films are deposited on a silicon wafer and are therefore an integral component of the device. The advantages of this process are that the sample has a uniform cross-sectional area, which removes the problem of the wedge-shaped geometry; it is an intrinsic component of the device, which eliminates attachment problems; and the geometry makes the system amenable to modeling. The primary disadvantage is that the metal films have to be deposited on a silicon wafer and then released.

The form of the stress-strain curve for the Al films investigated here, not shown due to space restrictions, is consistent with that from tests on larger samples: the elastic region is followed by a region in which the work hardening rate is high and then by a region of lower rate. The fracture morphology of a $500 \mathrm{~nm}$ thick sample is shown in Figure 2. The fracture surface is at $30^{\circ}$ to the tensile axis, extensive deformation occurs in all grains along the fracture surface and in grains up to $200 \mathrm{~nm}$ from the surface, and SEM images of the fracture surface shows a knife edge fracture perpendicular to the thickness direction. This shear band fracture is consistent with the material having limited working hardening capacity and low ductility.[9]

This example demonstrates that in situ studies of metal deformation can be done under known loading conditions by fabricating the experimental module using microlithographic techniques. 
References

1. S. Cheng, et al., Acta Mater. 53, 1521 (2005).

2. Q. Wei, et al., Mater.Sci. and Engr. A 381, 71 (2004).

3. D. Wolf, et al., Acta Mater. 53, 1 (2005).

4. K. S. Kumar, et al., Acta Mater. 51, 5743 (2003).

5. K. S. Kumar, et al., Acta Mater. 51, 387 (2003).

6. Z. Shan, et al., Science 305, 654 (2004).

7. M. A. Haque and M. T. A. Saif, Scripta Mater. 47, 863 (2002).

8. K. Hattar, et al., J. Mater. Res. 20, 1869 (2005).

9. This research was supported by a grant from award \# 02037400 and by the Center for Intergrated Nanotechnologies in Albuquerque, NM. The electron microscopy was performed in the Center for Microanalysis of Materials, University of Illinois at Urbana-Champaign, which is partially supported by the U.S. Department of Energy under grant DEFG02-91-ER45439 and by the University of Illinois. This work is supported by the Division of Materials Sciences and Engineering, Office of Basic Energy Sciences, U.S. Department of Energy, and done under the auspices of the Center of Integrated Nano Technologies. Sandia is a multiprogram laboratory operated by Sandia Corporation, a Lockheed Martin Company, for the Department of Energy's National Nuclear Security Administration under Contract No. DE-AC04-94-AL-85000.
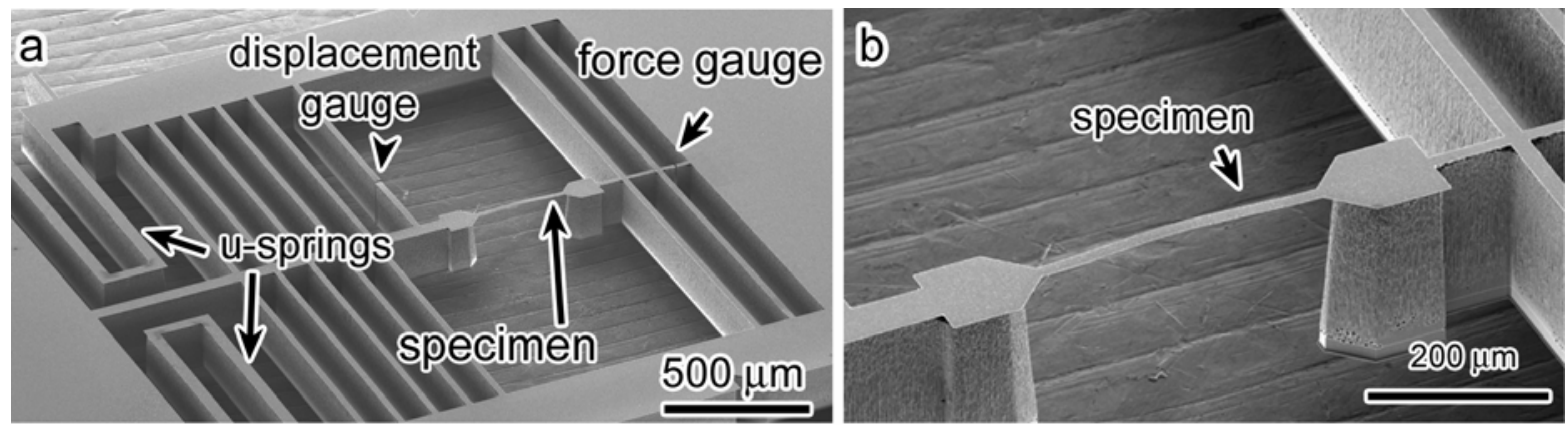

Figure 1. a) SEM image of fabricated device showing critical components, b) high magnification image of the tensile sample.


Figure 2. a) Shear fracture, b) bright-field TEM of fracture area,: insets show the different contrast from the indicated areas, c) SEM image of the knife-edge fracture surface of 500nm thick Al. 